\title{
Sexual Practices and HIV Prevalence amongst Men Who Have Sex with Men at a Community-Based Voluntary Counseling and Testing Centre in Malaysia
}

\author{
K. C. Koh, ${ }^{1}$ K. Kanagalingam, ${ }^{2}$ F. T. Tai, ${ }^{2}$ and A. Kamarulzaman ${ }^{3}$ \\ ${ }^{1}$ Department of Medicine, Clinical School, International Medical University, Jalan Rasah, Negeri Sembilan, \\ 70400 Seremban, Malaysia \\ ${ }^{2}$ PT Foundation, 7C-1, Jalan Ipoh Kecil, Off Jalan Raja Laut, P.O. Box 11859, 50350 Kuala Lumpur, Malaysia \\ ${ }^{3}$ The Center of Excellence for Research in AIDS (CERiA), University of Malaya Infectious Diseases Unit, \\ University of Malaya Medical Centre, Lembah Pantai, 59100 Kuala Lumpur, Malaysia
}

Correspondence should be addressed to K. C. Koh; kweechoy_koh@imu.edu.my

Received 15 November 2012; Accepted 5 December 2012

Academic Editors: B. Best and J. Ogwal-Okeng

Copyright $\odot 2013$ K. C. Koh et al. This is an open access article distributed under the Creative Commons Attribution License, which permits unrestricted use, distribution, and reproduction in any medium, provided the original work is properly cited.

\begin{abstract}
We describe the sexual practices and condom usage of men who have sex with men (MSM) at a community-based anonymous voluntary counseling and testing centre in Kuala Lumpur, Malaysia. This study is a first for Malaysia in this context. 433 MSM clients disclosed their sexual practices and condom use in the preceding 6 months using a self-reported questionnaire during preHIV test counseling at the centre. The mean age was 29.7 years, and 356 were homosexuals while 77 were bisexuals. Forty tested HIV positive (9.2\%). 387 (94.9\%) of 408 clients had anal sex, 395 (97.8\%) of 404 clients had oral sex, while 43 (18.4\%) of 233 clients had vaginal sex which revealed that even men who identified themselves as homosexuals do practice vaginal sex. Having multiple sexual partners is common (mean 11.6 partners per client). 259 (59.8\%) had unprotected sex within the last 6 months. Consistent condom use rates during vaginal, anal, and oral sex were $20 \%, 23.5 \%$, and $1.3 \%$, respectively. The odds ratio of testing HIV positive with inconsistent condom use during anal sex was $3.7(P=0.024)$. Clients who used condoms inconsistently during anal sex are more likely to be HIV positive.
\end{abstract}

\section{Introduction}

Men who have sex with men (MSM) are male persons who engage in sexual activity with members of the same sex, regardless of how they identify themselves. The term was created in the 1990s by epidemiologists in order to study the spread of disease among men who have sex with men, regardless of identity. Many men who engaged in sex with other men often do not consider themselves to be either gay or bisexual [1].

While the HIV epidemic seems to have stabilized at low levels in the general population or even declined globally over the last few years, amongst the MSM community, there is evidence of resurgence of HIV infection in the Western world as well as countries in Asia, Africa, and Latin America [27]. The reasons for the rise in HIV infection in MSM include high rates of unsafe sex, a new generation of young MSM unexposed to the devastating effects of HIV/AIDS in the early years of the epidemic, a shift of perception of HIV/AIDS from a terminal illness to a chronic treatable condition, lack of awareness of HIV infection status, infrequent testing by MSM, and independent risk factors for HIV acquisition such as substance abuse and alcohol use [2].

Between 2002 and 2007, HIV infection in several Asian countries such as Hong Kong and Taiwan increased by more than $200 \%$ [8, 9]. In the same period, Singapore and Japan reported a 4-fold and doubling of new HIV infections among MSM, respectively $[10,11]$.

Until recently, little is known about condom use during sexual intercourse and sexual habits of MSM in Malaysia. A survey using venue-day-time sampling by Kanter et al. [12] reported that MSM in Kuala Lumpur, Malaysia, have low 
knowledge of HIV transmission and engaged in high-risk sexual behaviour. We describe the prevalence of condom use during sexual intercourse, sexual habits, and HIV prevalence amongst MSM clients who sought HIV testing services at a community-based anonymous voluntary counseling and testing centre (VCT) in Kuala Lumpur, Malaysia.

\section{Materials and Methods}

Data were collated from the pre-HIV testing questionnaires filled by all clients seeking VCT services at the communitybased VCT centre from January 2008 until December 2008. Completion of the form was voluntary, and no data identifiable to the client were required. A total of 740 clients sought VCT services at the centre between Januaryl, 2008, and December 31, 2008. There were 433 clients who disclosed their sexuality as either homosexual or bisexual men and they were collectively categorized as MSM in this study. Transgender people have been excluded from this study as they are increasingly viewed as a separate group with unique needs and concerns [13].

Clients were asked to disclose their sexual practices, number of sexual partners in the preceding 6 months, last unprotected sexual intercourse, and condom usage during sexual intercourse in the preceding 6 months. Condom use during sexual intercourse was categorized as "consistent condom use" (condom was used on every single sexual intercourse in the preceding 6 months) and "inconsistent condom use" (condom may or may not have been used on every single sexual intercourse in the preceding 6 months).

HIV rapid tests were performed using either SD Bioline HIV test (Standard Diagnostics Inc.) or ACON HIV test kits according to the manufacturer's specification. Descriptive analysis including calculation of chi square and odds ratio was performed using SPSS for Windows v17. $P$ value of $<0.05$ was deemed significant. This study was approved by the National Medical Research Register of Malaysia.

\section{Results}

Of 433 MSM, 356 (82.2\%) identified themselves as homosexuals while $77(17.8 \%)$ identified themselves as bisexuals. The mean age was 29.2 years old (median 28 years). The youngest was 18 years old, while the oldest was 61 years old. Most of the clients were of Chinese $(N=253)$ and Malays $(N=115)$ ethnic groups. Most of the clients were unmarried (310) and were not in a relationship (340) at the time of testing. The other sociodemographic parameters are tabulated in Table 1. Forty clients $(9.2 \%)$ tested HIV positive using the HIV rapid test kits. Of these, 32 were homosexuals and 8 were bisexuals.

In the preceding 6 months, 387 clients reported having engaged in anal sex, 395 in oral sex, and 43 in vaginal sex (Table 2). In terms of combination of sexual practices (Table $3)$, the majority $(N=337)$ have engaged in both anal and oral sex, 25 engaged in oral, vaginal, and anal sex, 10 engaged in oral and vaginal sex, 22 engaged in oral sex only, 23 engaged in anal sex only, and 6 engaged in vaginal sex only.
TABLE 1: Sociodemographic profiles of MSM seeking VCT services.

\begin{tabular}{lcc}
\hline Sociodemographic factors & Frequency & Percentage \\
\hline Age groups in years $(N=431)^{*}$ & 7 & \\
$\quad<20$ & 258 & 1.6 \\
$20-29$ & 130 & 30.8 \\
$30-39$ & 28 & 6.5 \\
$40-49$ & 6 & 1.4 \\
$50-59$ & 2 & 0.5 \\
$>60$ & & \\
Ethnic group $(N=429)^{*}$ & 115 & 26.8 \\
$\quad$ Malay & 253 & 59.0 \\
Chinese & 21 & 4.9 \\
Indian & 40 & 9.3 \\
Other & & \\
Marital status $(N=318)^{*}$ & 310 & 97.5 \\
Single & 8 & 2.5 \\
Married & & \\
Current relationship status $(N=421)^{*}$ & 340 & 80.8 \\
Not in relationship & 55 & 13.1 \\
Monogamous relationship & 26 & 6.1 \\
Open relationship & & \\
Sexuality $(N=433)^{*}$ & 356 & 82.2 \\
Homosexual & 77 & 17.8 \\
Bisexual & & \\
* & & \\
\hline
\end{tabular}

* Number of clients who responded to this part of the pre-HIV testing questionnaire.

TABLE 2: Sexual practices engaged by MSM in the preceding 6 months.

\begin{tabular}{lc}
\hline Sexual practice & $\begin{array}{c}\text { Number engaged in } \\
\text { the sexual practice }\end{array}$ \\
\hline Vaginal sex $(N=233)^{*}$ & $15(8.0 \%)$ \\
$\quad$ (i) Homosexuals $(N=187)$ & $28(60.9 \%)$ \\
$\quad$ (ii) Bisexuals $(N=46)$ & \\
Oral sex $(N=404)^{*}$ & $328(97.9 \%)$ \\
(i) Homosexuals $(N=335)$ & $67(97.1 \%)$ \\
(ii) Bisexuals $(N=69)$ & \\
Anal sex $(N=408)^{*}$ & $322(95.0 \%)$ \\
(i) Homosexuals $(N=339)$ & $65(94.2 \%)$ \\
(ii) Bisexuals $(N=69)$ &
\end{tabular}

* Number of clients who responded to this part of the pre-HIV testing questionnaire.

With regards to number of sexual partners in the preceding 6 months (Table 4), 159 clients had less than 10 sexual partners, 59 had between 10 and 19 sex partners, 32 had between 20 and 29 sex partners, while 27 clients had more than 30 sex partners. The average number of sex partners per client in the preceding 6 months was 11.6 (median 6). The difference in the HIV prevalence amongst clients with less than 10 sexual partners in the preceding 6 months compared to clients who had more than 10 sexual partners was not statistically significant $(P=0.307)$. Within the preceding 6 
TABLE 3: Combinations of sexual practices amongst MSM.

\begin{tabular}{lcc}
\hline $\begin{array}{l}\text { Sexual practice } \\
\text { combinations }\end{array}$ & Homosexuals* & Bisexuals* $(\%)$ \\
\hline Vaginal only & $5(1.4 \%)$ & $1(1.4 \%)$ \\
Anal only & $20(5.7 \%)$ & $3(4.1 \%)$ \\
Oral only & $17(4.8 \%)$ & $5(6.8 \%)$ \\
Vaginal and anal & 0 & $2(2.7 \%)$ \\
Vaginal and oral & $7(2.0 \%)$ & $3(4.1 \%)$ \\
Anal and oral & $300(85.2 \%)$ & $37(50.7 \%)$ \\
Vaginal, anal, and oral & $3(0.9 \%)$ & $22(30.1 \%)$ \\
\hline Total & $352(100 \%)$ & $73(100 \%)$ \\
\hline
\end{tabular}

Number of clients who responded to this part of the pre-HIV testing questionnaire.

TABLE 4: Number of sexual partners in preceding last 6 months amongst MSM $(N=277)^{*}$ and their HIV test results.

\begin{tabular}{lcc}
\hline $\begin{array}{l}\text { Number of sex partners in } \\
\text { preceding 6 months }\end{array}$ & $N$ & HIV positive \\
\hline$<10$ & 159 & $15(9.4 \%)$ \\
$10-19$ & 59 & $5(8.5 \%)$ \\
$20-29$ & 32 & $4(12.5 \%)$ \\
$>30$ & 27 & $1(3.7 \%)$ \\
\hline Total & 277 & $25(9.0 \%)$ \\
${ }^{*}$ Number of clients who responded to this part of the pre-HIV testing \\
questionnaire.
\end{tabular}

TABLE 5: Condom use during sexual intercourse amongst MSM and HIV test results.

\begin{tabular}{lcc}
\hline Sexual practices & $N(\%)$ & HIV positive (\%) \\
\hline Vaginal sex $(N=40)^{*}$ & & \\
$\quad$ (i) Consistent condom use & $8(20 \%)$ & 0 \\
$\quad$ (ii) Inconsistent condom use & $32(80 \%)$ & $4(12.5 \%)$ \\
Oral sex $(N=234)^{*}$ & & \\
$\quad$ (i) Consistent condom use & $3(1.3 \%)$ & 0 \\
$\quad$ (ii) Inconsistent condom use & $231(98.7 \%)$ & $21(9.1 \%)$ \\
Anal sex $(N=371)^{*}$ & \\
$\quad$ (i) Consistent condom use & $87(23.5 \%)$ & $3(3.4 \%)$ \\
$\quad$ (ii) Inconsistent condom use & $284(76.5 \%)$ & $33(11.6 \%)$ \\
\hline
\end{tabular}

"Number of clients who responded to this part of the pre-HIV testing questionnaire.

months, 259 (59.8\%) clients disclosed that they had engaged in unprotected sexual intercourse.

With regards to condom use during vaginal sex in the preceding 6 months, $32(80.0 \%)$ out of 40 clients reported inconsistent condom (Table 5). Of these 32 clients, 4 (12.5\%) tested positive for HIV. Two hundred thirty-one (98.7\%) out of 234 clients reported inconsistent condom use during oral sex, of which $21(9.1 \%)$ tested positive for HIV. Two hundred eighty-four (76.5\%) out of 371 clients reported the inconsistent condom use during anal sex, of which 33 (11.6\%) clients tested positive for HIV. Interestingly, 3 clients who claimed to use condoms consistently during anal sex also tested positive for HIV.

Clients who used condoms inconsistently during anal sex were 3.7 times more likely to be HIV positive $(P=0.024)$. Statistical significance was not found between inconsistent condom use during oral or vaginal sex and HIV positivity $(P>0.05)$. In our study, sexual practice and the number of sex partners in preceding 6 months were not associated with higher HIV prevalence $(P>0.05)$.

\section{Discussion}

The HIV epidemic in Asia has traditionally been fueled and concentrated in high-risk populations, namely, the injecting drug users, sex workers and their clients, and MSM. From these 3 groups, HIV is steadily expanding into lower-risk populations through transmission to the sexual partners of those most at risk [14].

The prevalence of male-to-male sex in 2008 in Southeast Asia was estimated to be between $6 \%$ and 12\% [15]. Between 2005 and 2008, the MSM populations in several Southeast Asian countries have been estimated to be 766,000 in Indonesia, 240,000 in Myanmar, and 560,000 in Thailand [13]. In Singapore, the MSM population makes up between $5 \%$ and $10 \%$ of the total population (Tan CK, PT Foundation personal communication).

In a review of cross-sectional studies done in Asian countries, the HIV prevalence in MSM ranged $0.7-8.7 \%$ in Cambodia, $0-7.8 \%$ in Vietnam, $17.3-30.8 \%$ in Thailand, 5.6\% in Lao PDR, 23.5\% in Yangon (Myanmar), 35\% in Mandalay (Myanmar), $8.5 \%$ in Taipei, $4.2 \%$ in Singapore, $4.1 \%$ in Hong Kong, and between $2 \%$ and $8.1 \%$ in the Indonesian cities of Bandung, Surabaya, and Jakarta, respectively [12-16].

In Malaysia, the actual prevalence of HIV or risk behaviors amongst MSM is unknown. A recent venue-based study in Kuala Lumpur amongst MSM by Kanter [12] reported the HIV prevalence to be $3.9 \%$. The Malaysian Ministry of Health National AIDS Surveillance Report from 1986 to December 2007 indicated that $1.8 \%$ of 80,938 and $3.08 \%$ of 13,635 cumulative HIV and AIDS cases, respectively, were infected through homosexual or bisexual activities [17].

In our study cohort, the HIV prevalence was $9.2 \%$. This figure is higher than what was reported by Kanter [12]. This is unsurprising as HIV prevalence estimation based on VCT data is dependent on a number of factors such as changes in test-seeking behavior, attitudes, and behaviours toward VCT acceptability and participation bias. Generally, HIV prevalence based on VCT data would expectedly be higher than the prevalence in the general population $[18,19]$.

The majority of the clients in our study engaged in a combination of oral and anal sex while a number of them also engaged in vaginal sex although these men still identified themselves as homosexuals (Table 2). It is not unusual for MSM to be married or have female sex partners as reported in a number of studies [13]. This small but significant group of men who have both male and female sexual partners may be the outlet conduit for HIV infection to spread from the 
MSM population into the heterosexual population, namely, through their female spouses and sex partners.

The majority of the clients in our study had multiple sexual partners. In the MSM community, having multiple sexual partners is well recognised. In the WHO SEARO [13] report, a substantial proportion of MSM had large numbers of male sex partners of all types-regular, casual, and commercial (paid) and paying. The mean number of male sex partners ranged from 1.7 to 13.9 over one month in India (2006), 3.9 over one month in Bangladesh (20032004), and 8.8 over 12 months in Sri Lanka (2006-2007). In Indonesia, the median number of male sex partners of MSM over one month ranged between 2 and 10 [13].

In our study, the average number of sexual partners per client in the preceding 6 months was 11.6. Interestingly, there was no statistically significant difference in the prevalence of HIV between those who had less than 10 sexual partners and those who had more than 10 sexual partners $(P>0.05)$. While having multiple sexual partners is often regarded as a risk factor for HIV acquisition, it was not the case here. A number of studies have demonstrated that factors, besides the number of sexual partners, are also significant independent as well as collective risk factors in fueling the HIV epidemic. These factors include the number of concurrent sexual partners rather than consecutive sexual partners, concurrent use of alcohol, recreational drugs, injecting drug use, previous history of other sexually transmitted infections (STI), and inconsistent condom use as well as sexual behavior during intercourse $[20,21]$.

Correct and consistent condom use during sexual intercourse remains the most effective method of preventing HIV transmission $[22,23]$. In the Thai " $100 \%$ Condom Use Programme" in the 1990s, condom use was aggressively promoted in sex work venues, resulting in increased condom use in these settings and decline of five major sexually transmitted diseases in men by $79 \%$, thus demonstrating the efficacy of condom usage. When condom use among clients of sex workers reached $>80 \%$, transmission of STI and HIV decreased markedly [24].

Unfortunately, the rate of condom use by MSM in Southeast Asia countries is generally very low. The United Nations Economic and Social Commission for Asia and the Pacific (UNESCAP) in its report for 2009 revealed that while $>80 \%$ of MSM in Thailand and Cambodia used condoms, other Southeast Asian countries have much lower rate of condom use. For instance, only $24.2 \%$ of MSM in Lao PDR, $32 \%$ of MSM in the Philippines, and 39.3\% of MSM in Indonesia used condoms [25]. A study in Yunnan, China, reported that men who do not use condoms with their MSM partners were more likely not to use condoms with their female partners as well [26].

Until now, the rate of condom use during amongst MSM in Malaysia is unknown. The high rates of inconsistent condom use disclosed by clients in this study are alarming with $80 \%$ not using a condom consistently during vaginal sex, $98.7 \%$ during oral sex, and $76.5 \%$ during anal sex, respectively (Table 5).

None of those who reported using condom consistently during oral $(N=3)$ and vaginal sex $(N=8)$ tested positive for HIV. Unfortunately, 3 out of 87 clients who reported consistent condom use during anal sex tested positive for HIV. None of these 3 clients reported engaging in vaginal sex although one of them reported inconsistent condom use during oral sex while the remaining 2 clients did not disclose if they had also engaged in oral sex with or without condom use. Likewise, 21 out of 231 clients who reported inconsistent condom use during oral sex also tested HIV positive. Many MSM perceive oral sex to be "safe" and relatively risk-free in terms of acquiring HIV infection, and therefore it was not surprising that the rate of condom use during oral sex was very low in our study. Although the use of condom during sex has been publicized as an effective preventive method against transmission of HIV, it is not infallible. In addition, other factors may have contributed to HIV transmission in these settings including the incorrect use of condoms, engaging but failing to report in other risky sexual practices, engaging in unprotected anal or vaginal sex beyond the preceding 6 months, and the sexual roles during sex (i.e., as a inserter or receiver) $[22,23]$.

The low rate of consistent condom use during anal sex in our study cohort is a cause for grave concern. Unprotected anal sex has the highest risk of HIV transmission because the thin lining of the rectum and anus can easily be compromised during sex. In our study, the odds ratio of acquiring HIV infection for a person who engaged in anal sex with inconsistent condom use is 3.7 times higher compared to a person who consistently used condoms during anal sex. The possible reasons contributing to inconsistent condom use may be the relative inaccessibility of condoms at venues of sexual activities, ignorance or lack of knowledge in the spread of sexually transmitted infections, incorrect perception of one's own likelihood of HIV acquisition, concurrent substance abuse, the false security in having a steady partner, lack of communication with partners regarding risk reduction practices, frequent rough intercourse, as well as attitudinal and behavioral issues. [27]. In Kanter et al.'s [12] study on a similar MSM population in Malaysia, one in five participants did not believe that HIV could be transmitted through insertive or receptive anal sex.

\section{Conclusion}

Our study showed that the sexual practices of MSM in our study cohort are not unlike their counterparts in other Asian countries. A significant number of clients in our study cohort also have female sex partners, potentially becoming a conduit for the spread of HIV to the non-MSM population as is happening in other parts of Asia. The low rate of consistent condom use during anal sex in our study cohort is also a cause for concern.

Our findings in this study were subjected to 3 potential biases. First, our findings would be biased if participants chose not to give accurate information regarding their sexual practices, condom use, or whether they had tested HIV positive previously in another testing facility. We did not use computer-based self-administered questionnaire which may be more effective in obtaining sensitive information [28]. 
Perceived social expectations may also give rise to under reporting of sexual risk behavior. We observed no association between sexual practices, number of sexual partners, and last unprotected sex and HIV prevalence in our study.

Secondly, since our study was confined to MSM who attended a MSM-identified VCT centre, our findings may not be generalized to MSM who do not use VCT services. Thirdly, MSM requesting VCT services have high-risk sexual behaviors and are at risk of HIV acquisition. We observed a higher HIV prevalence amongst MSM in our study compared to a venue-based study conducted in the same city [12].

Nevertheless, our findings of high-risk sexual behaviors coupled with a high rate of inconsistent condom use are major concerns. Specific interventions that target sexual risk behaviors and condom use are necessary in this group.

\section{Conflict of Interests}

The authors declare no conflict of interests in submitting this paper for publication. They further declare that this study received no funding from any organizations or persons.

\section{Authors' Contributions}

K. C. Koh contributed to the study design, data collating and data entry, statistical analysis, literature search, and writing of the paper. A. Kamarulzaman helped with the Study design and reviewing of the paper. K. Kanagalingam and F. T. Tai contributed to the formulation of questionnaire and data gathering.

\section{References}

[1] H. Blanton and M. Gerrard, "Effect of sexual motivation on men's risk perception for sexually transmitted disease: there must be 50 ways to justify a lover," Health Psychology, vol. 16, no. 4, pp. 374-379, 1997.

[2] H. W. Jaffe, R. O. Valdiserri, and K. M. De Cock, "The reemerging HIV/AIDS epidemic in men who have sex with men," Journal of the American Medical Association, vol. 298, no. 20, pp. 2412-2414, 2007.

[3] A. E. Grulich and J. M. Kaldor, "Trends in HIV incidence in homosexual men in developed countries," Sexual Health, vol. 5, no. 2, pp. 113-118, 2008.

[4] G. Likatavičius, I. Klavs, I. Devaux, J. Alix, and A. Nardone, "An increase in newly diagnosed HIV cases reported among men who have sex with men in Europe, 2000-6: implications for a European public health strategy," Sexually Transmitted Infections, vol. 84, no. 6, pp. 499-505, 2008.

[5] J. W. D. Lind Van Wijngaarden, T. Brown, P. Girault, S. Sarkar, and F. Van Griensven, "The epidemiology of human immunodeficiency virus infection, sexually transmitted infections, and associated risk behaviors among men who have sex with men in the mekong subregion and China: Implications for policy and programming," Sexually Transmitted Diseases, vol. 36, no. 5, pp. 319-324, 2009.

[6] F. Van Griensven, "Men who have sex with men and their HIV epidemics in Africa," AIDS, vol. 21, no. 10, pp. 1361-1362, 2007.

[7] C. F. Cáceres, K. Konda, E. R. Segura, and R. Lyerla, "Epidemiology of male same-sex behaviour and associated sexual health indicators in low- and middle-income countries: 2003-2007 Estimates," Sexually Transmitted Infections, vol. 84, supplement 1, pp. i49-i56, 2008.

[8] Center for Health Protection, Department of Health. Hong Kong: AIDS Office. Virtual AIDS Office of Hong Kong, 2010, http://www.info.gov.hk/aids/english/surveillance/off_surreport .htm.

[9] Centers for Disease Control, Ministry of Health, Taipei, Taiwan, 2008, http://www.cdc.gov.tw/english/list.aspx?treeid =00ED75D6C887BB27\&nowtreeid $=$ A6C722111C023FC2.

[10] Ministry of Health Singapore, Republic of Singapore, September 2008, http://www.moh.gov.sg/content/dam/moh_web/ PressRoom/Articles/2012/Annex_HIV\%20Figures\%202011 .pdf.

[11] National Institute of Infectious Diseases, Ministry of Health, Labour and Welfare, Tokyo, Japan, June 2008, http://idsc .nih.go.jp/iasr/29/340/tpc340.html.

[12] J. Kanter, C. Koh, K. Razali et al., "Risk behaviour and HIV prevalence among men who have sex with men in a multiethnic society: a venue-based study in Kuala Lumpur, Malaysia," International Journal of STD and AIDS, vol. 22, no. 1, pp. 30-37, 2011.

[13] WHO SEARO, "HIV/AIDS among men who have sex with men and transgender populations in South-East Asia," The current situation and national responses, 2010.

[14] WHO, HIV/AIDS in the South-East Asia Region, 2009.

[15] C. Cáceres, K. Konda, M. Pecheny, A. Chatterjee, and R. Lyerla, "Estimating the number of men who have sex with men in low and middle income countries," Sexually Transmitted Infections, vol. 82, supplement 3, pp. iii3-iii9, 2006.

[16] F. Van Griensven, J. W. D. L. Van Wijngaarden, S. Baral, and A. Grulich, "The global epidemic of HIV infection among men who have sex with men," Current Opinion in HIV and AIDS, vol. 4, no. 4, pp. 300-307, 2009.

[17] Ministry of Health Malaysia, STI Unit Resource Centre, Malaysian AIDS Council, 1986-December 2007.

[18] W. McFarland, M. P. Busch, T. A. Kellogg et al., "Detection of early HIV infection and estimation of incidence using a sensitive/less-sensitive enzyme immunoassay testing strategy at anonymous counseling and testing sites in San Francisco," Journal of Acquired Immune Deficiency Syndromes and Human Retrovirology, vol. 22, no. 5, pp. 484-489, 1999.

[19] N. Meda, M. T. Zoundi-Guigui, P. Van de Perre et al., "HIV infection among pregnant women in Bobo-Dioulasso, Burkina Faso: comparison of voluntary and blinded seroprevalence estimates," International Journal of STD and AIDS, vol. 10, no. 11, pp. 738-740, 1999.

[20] M. Morris, H. Epstein, and M. Wawer, “Timing is Everything: international variations in historical sexual partnership concurrency and HIV prevalence," PLoS ONE, vol. 5, no. 11, Article ID e14092, 2010.

[21] B. A. Koblin, M. J. Husnik, G. Colfax et al., "Risk factors for HIV infection among men who have sex with men," AIDS, vol. 20, no. 5, pp. 731-739, 2006.

[22] M. Tanaka, H. Nakayama, M. Sakumoto, T. Matsumoto, K. Akazawa, and J. Kumazawa, "Trends in sexually transmitted diseases and condom use patterns among commercial sex workers in Fukuoka City, Japan 1990-93," Sexually Transmitted Infections, vol. 72, no. 5, pp. 358-361, 1996.

[23] W. L. Roper, H. B. Peterson, and J. W. Curran, "Commentary: condoms and HIV/STD prevention-clarifying the message," 
American Journal of Public Health, vol. 83, no. 4, pp. 501-503, 1993.

[24] R. S. Hanenberg, W. Rojanapithayakorn, P. Kunasol, and D. C. Sokal, "Impact of Thailand's HIV-control programme as indicated by the decline of sexually transmitted diseases," The Lancet, vol. 344, no. 8917, pp. 243-245, 1994.

[25] UNESCAP, Statistical Yearbook for Asia and in the Pacific 2009, http://www.unescap.org/stat/data/syb2009/7-HIV-andAIDS.pdf.

[26] J. T. F. Lau, M. Wang, H. N. Wong et al., "Prevalence of bisexual behaviors among men who have sex with men (MSM) in China and associations between condom use in MSM and heterosexual behaviors," Sexually Transmitted Diseases, vol. 35, no. 4, pp. 406-413, 2008.

[27] G. Remafedi, "Predictors of unprotected intercourse among gay and bisexual youth: knowledge, beliefs, and behavior," Pediatrics, vol. 94, no. 2 I, pp. 163-168, 1994.

[28] C. F. Turner, L. Ku, S. M. Rogers, L. D. Lindberg, J. H. Pleck, and F. L. Sonenstein, "Adolescent sexual behavior, drug use, and violence: increased reporting with computer survey technology," Science, vol. 280, no. 5365, pp. 867-873, 1998. 


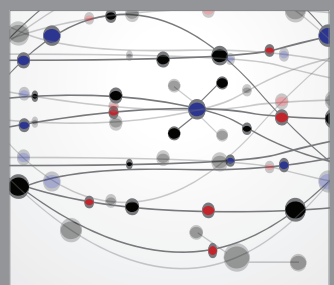

The Scientific World Journal
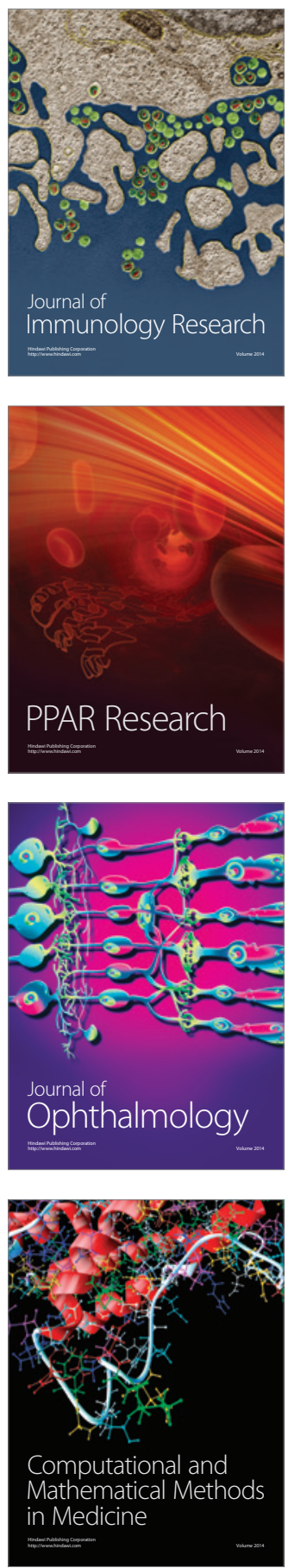

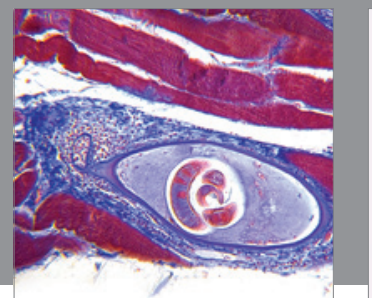

Gastroenterology

Research and Practice
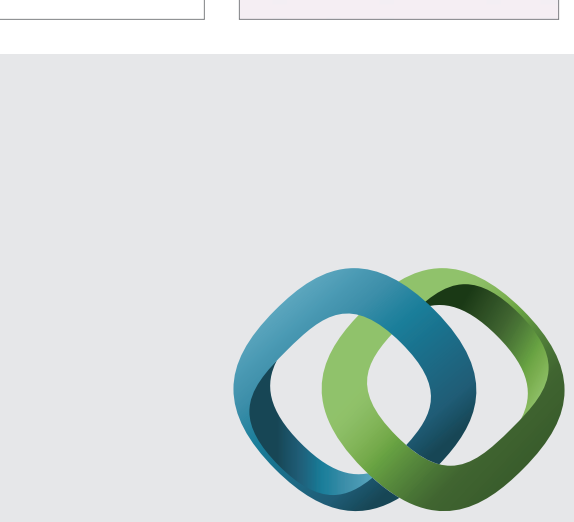

\section{Hindawi}

Submit your manuscripts at

http://www.hindawi.com
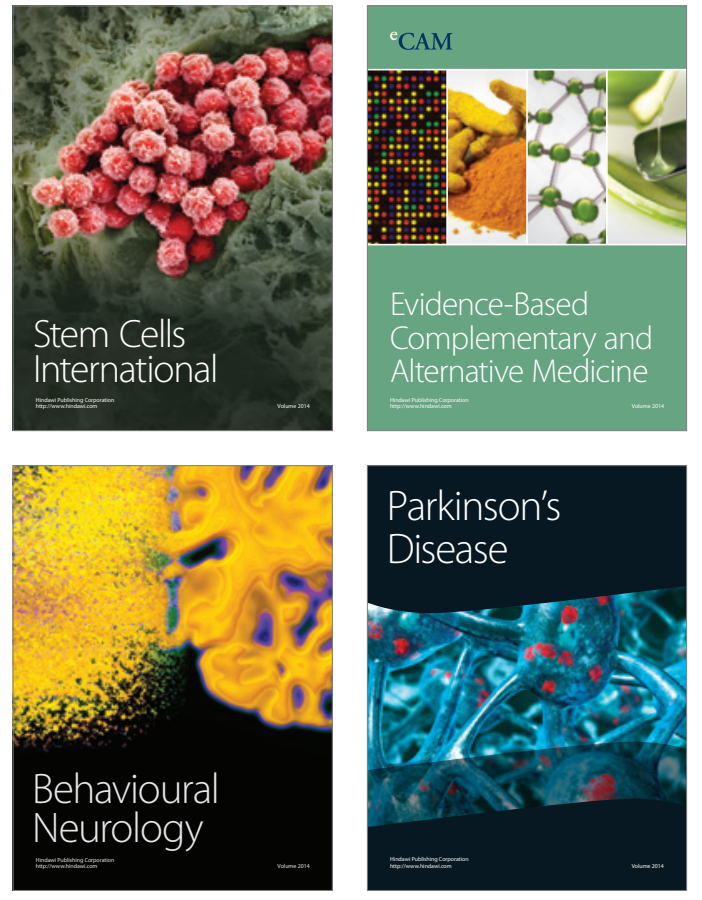
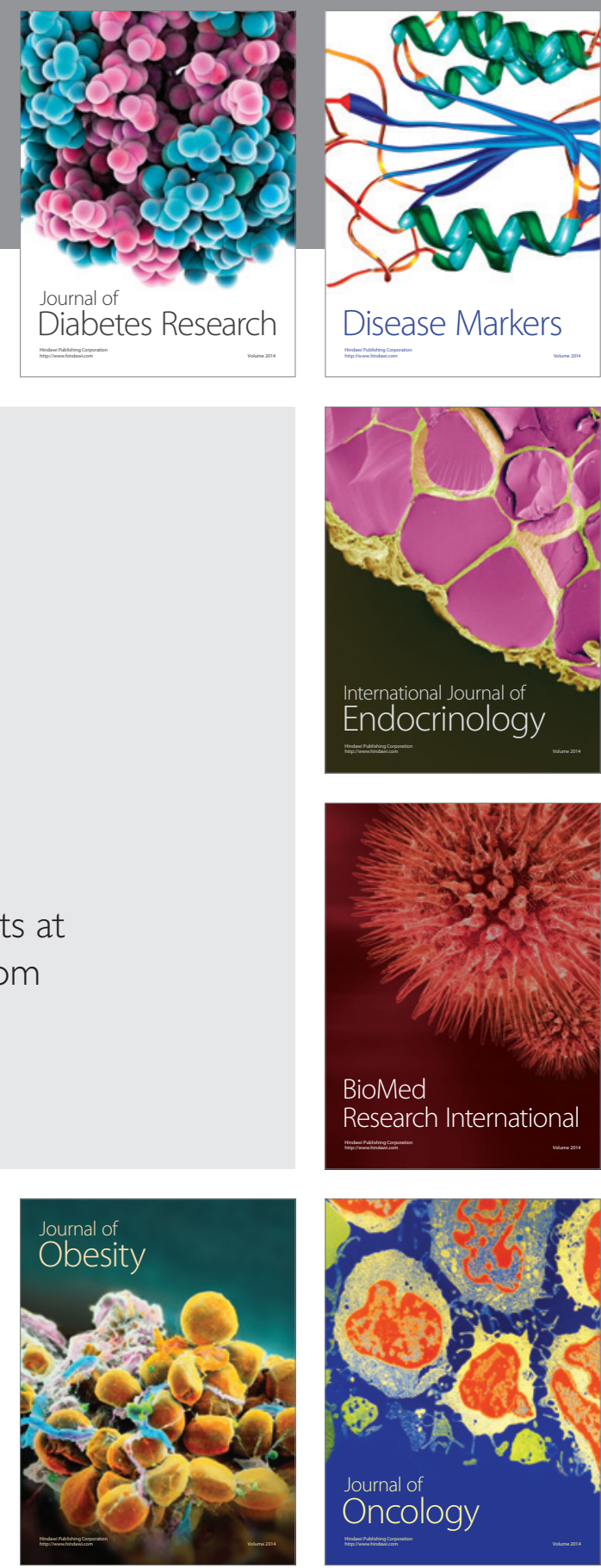

Disease Markers
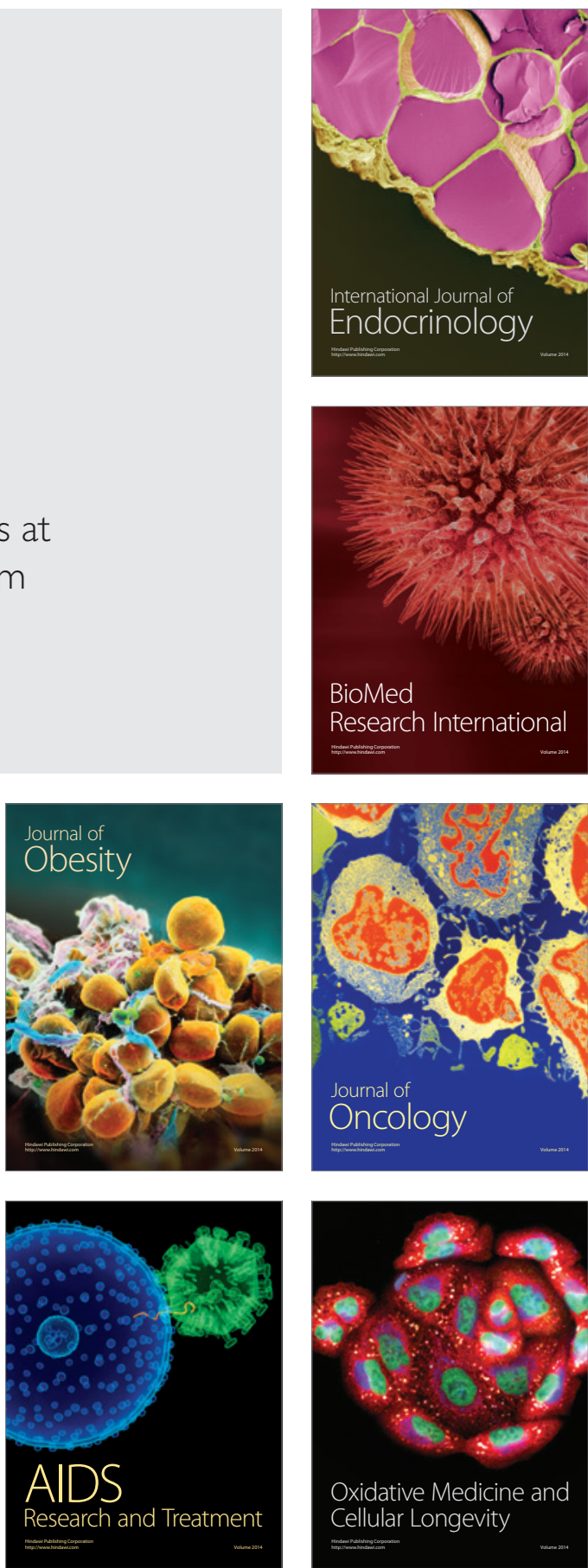